\title{
Combined Parameter and State Estimation Algorithms for Multivariable Nonlinear Systems Using MIMO Wiener Models
}

\author{
Houda Salhi and Samira Kamoun \\ University of Sfax, National Engineering School of Sfax (ENIS), Laboratory of Sciences and Technique of \\ Automatic Control and Computer Engineering (Lab-SAT), BP 1173, 3038 Sfax, Tunisia \\ Correspondence should be addressed to Houda Salhi; salhi.houda85@yahoo.fr
}

Received 29 November 2015; Revised 20 April 2016; Accepted 12 May 2016

Academic Editor: James Lam

Copyright ( $\odot 2016$ H. Salhi and S. Kamoun. This is an open access article distributed under the Creative Commons Attribution License, which permits unrestricted use, distribution, and reproduction in any medium, provided the original work is properly cited.

\begin{abstract}
This paper deals with the parameter estimation problem for multivariable nonlinear systems described by MIMO state-space Wiener models. Recursive parameters and state estimation algorithms are presented using the least squares technique, the adjustable model, and the Kalman filter theory. The basic idea is to estimate jointly the parameters, the state vector, and the internal variables of MIMO Wiener models based on a specific decomposition technique to extract the internal vector and avoid problems related to invertibility assumption. The effectiveness of the proposed algorithms is shown by an illustrative simulation example.
\end{abstract}

\section{Introduction}

Over the last years, modeling, identification, and parameter estimation theories have received much attention by various research teams [1-4]. Blocks-oriented nonlinear models, in particular, which consist of interconnected linear dynamic subsystems and memory less nonlinear elements, have been widely used for modeling a large variety of nonlinear systems in such different fields as mechanical dynamics [5], chemical process [6], biotechnologies [7], signal filtering [8], and so on. In fact, this class of nonlinear models is able to describe the dynamic of complex systems, with a relatively simple structure. They can even simplify the identification, control, or diagnostic problems [9-12]. Added to that, several approaches developed in the linear case can be applied with an appropriate practical implementations [13-19]. In recent years, many identification methods have been studied for blocks-oriented systems and a large amount of works have been published in the literature. For example, Vörös [20] proposed a least squares based iterative algorithm for Hammerstein-Wiener systems with a backlash output; $\mathrm{Hu}$ et al. [21] developed an extended least squares parameter estimation algorithm for Wiener systems based on the overparameterization method; Ding et al. [22] used the hierarchical identification principal to identify Hammerstein systems; Mao and Ding [8] proposed a multi-innovation stochastic gradient algorithm for Hammerstein systems using the key term separation principal; Li [23] studied the maximum likelihood estimation algorithm for Hammerstein CARARMA systems; Guo and Bretthauer [24] proposed a recursive identification method for Wiener models, based on the prediction error method. Furthermore, Chaudhary et al. [25] explored an adaptive algorithm based on fractional signal processing for parameter estimation of Hammerstein autoregressive models; Wu et al. [26] presented a robust Hammerstein adaptive filtering algorithm based on the Maximum Correntropy Criterion, which aims at maximizing the similarity between the model output and the reel response; Falck et al. [27] proposed an identification method of NARX Wiener-Hammerstein models using kernel-based estimation technique; Kibangou and Favier [28] developed a new approach for estimating a Parallel-Cascade Wiener System using a joint diagonalization of the $p$ th-order Volterra kernel slices to identify linear zsubsystems and using the least square algorithm to identify nonlinear subsystems. This approach has been extended to other blocks-oriented models with polynomials nonlinearities [29].

Recently, much attention has been paid to blocksoriented state-space systems which have been successfully 
used for control algorithms, identification schemes, and signal filtering [30, 31]. However, the parameter estimation has become more difficult because the blocks-oriented models not only include the unknown parameter of linear and nonlinear subsystems but also include the unmeasurable state variables [32-35]. In this framework, Wang and Ding proposed a recursive parameter and state estimation for Hammerstein state-space systems [36] and for HammersteinWiener state-space systems [37], using the hierarchical principal; Wang et al. [38] discussed an iterative identification algorithm Hammetstein state-space system, by combining the iterative least square and the hierarchical identification method. However, for Wiener state-space models, there is a little contribution in the literature that addresses the parameter estimation problems or the state estimation problems. In fact, Westwick and Verhaegen [39] proposed a subspace identification method for MIMO Wiener subsystems with odd and even nonlinearities and a Gaussian input system; Bruls et al. [40] derived separable least squares algorithms for a state-space Wiener model with Chebyshev polynomials nonlinearity; Lovera et al. [41] developed a recursive subspace identification method for Wiener state-space models using the singular-value decomposition technique; Glaria Lopez and Sbarbaro [42] proposed an observer design for a Wiener model with known parameters.

The main difficulty in the identification of Wiener models is that the internal variables, acting between linear and nonlinear blocks, are almost unavailable and the input-output available data are not enough to provide all information on these unknown variables. To overcome this difficulty, most published works, addressing the identification of Wiener systems, assume one of these assumptions: the invertibility of the unknown nonlinear element [43], an a priori knowledge of the nonlinearity [44], an approximation of the nonlinearity as a piecewise linear function [44], and a specific input signal [39]. However, these assumptions, and especially the invertibility assumption, severely limit the applicability of Wiener models because the output nonlinearity, in several real cases, is noninvertible or is quite complicated to find the inverse nonlinearity especially for multivariable systems.

This paper introduces a recursive identification method for MIMO Wiener model. This model is characterised by a linear dynamic block as an observer state-space model and a nonlinear block as combined and arbitrary (reversible or irreversible) nonlinearities. A recursive algorithm which combines the least squares technique, the adjustable model, and the Kalman filter principle is developed to resolve the parameters and state estimation problem with less computational effort and a fast convergence rate. Indeed, in the proposed method, the parameters of the linear part and nonlinear part of the MIMO Wiener model are estimated separately in order to decrease the dimension of the unknown parameters matrices and reduce the parameter redundancy. Moreover, a modified Kalman filter and a specific decomposition technique are developed to extract and estimate the unknown internal vector without any research of the inverse nonlinear functions.

The remainder of this paper is organized as follows. Section 2 describes the problem formulation for MIMO Wiener state-space models. The least squares based and adjustable model based recursive parameter estimation algorithm and a new recursive state estimation algorithm based on Kalman filter theorem are presented in Section 3. Section 4 provides an illustrative example to show the efficiency of the proposed algorithms. Finally, some concluding remarks are given in Section 5.

\section{Problem Formulation}

Consider the MIMO discrete-time Wiener model Figure 1 where the linear dynamic part is given by the following statespace equation:

$$
\begin{aligned}
x(k+1) & =A(k) x(k)+B(k) U(k)+W(k) \\
Z(k) & =C(k) x(k)+D(k) U(k)+V(k),
\end{aligned}
$$

where $x^{T}(k)=\left[\begin{array}{llll}x_{1}(k) & x_{2}(k) & \cdots & x_{n_{X}}(k)\end{array}\right], \quad U^{T}(k)=$ $\left[\begin{array}{llll}u_{1}(k) & u_{2}(k) & \cdots & u_{n_{U}}(k)\end{array}\right], \quad$ and $Z^{T}(k)$ = $\left[\begin{array}{llll}z_{1}(k) & z_{2}(k) & \cdots & z_{n_{z}}(k)\end{array}\right]$ are, respectively, the state vector, the input vector, and the internal vector at the discrete-time $k, W^{T}(k)=\left[\begin{array}{llll}w_{1}(k) & \cdots & w_{n_{X}}(k)\end{array}\right]$ and $V^{T}(k)=$ $\left[\begin{array}{lll}v_{1}(k) & \cdots & v_{n_{Z}}(k)\end{array}\right]$ are two noise vectors, $A(k) \in R^{n_{X} \times n_{X}}$, $B(k) \in R^{n_{X} \times n_{U}}$, and $C(k) \in R^{n_{Z} \times n_{X}}$ and $D(k) \in R^{n_{Z} \times n_{U}}$ are defined, respectively, by

$$
\begin{aligned}
A(k) & =\left[\begin{array}{cccc}
a_{11}(k) & a_{12}(k) & \cdots & a_{1 n_{X}}(k) \\
a_{21}(k) & a_{22}(k) & \cdots & a_{2 n_{X}}(k) \\
\vdots & \vdots & \ddots & \vdots \\
a_{n_{X} 1}(k) & a_{n_{X} 2}(k) & \cdots & a_{n_{X} n_{X}}(k)
\end{array}\right] \\
B(k)= & {\left[\begin{array}{cccc}
b_{11}(k) & b_{12}(k) & \cdots & b_{1 n_{U}}(k) \\
b_{21}(k) & b_{22}(k) & \cdots & b_{2 n_{U}}(k) \\
\vdots & \vdots & \ddots & \vdots \\
b_{n_{X} 1}(k) & b_{n_{X} 2}(k) & \cdots & b_{n_{X} n_{U}}(k)
\end{array}\right] } \\
C(k)= & {\left[\begin{array}{cccc}
c_{11}(k) & c_{12}(k) & \cdots & c_{1 n_{X}}(k) \\
c_{21}(k) & c_{22}(k) & \cdots & c_{2 n_{X}}(k) \\
\vdots & \vdots & \ddots & \vdots \\
c_{n_{Z} 1}(k) & c_{n_{Z} 2}(k) & \cdots & c_{n_{Z} n_{X}}(k)
\end{array}\right] } \\
D(k)= & {\left[\begin{array}{cccc}
d_{11}(k) & d_{12}(k) & \cdots & d_{1 n_{U}}(k) \\
d_{21}(k) & d_{22}(k) & \cdots & d_{2 n_{U}}(k) \\
\vdots & \vdots & \ddots & \vdots \\
d_{n_{Z} 1}(k) & d_{n_{Z} 2}(k) & \cdots & d_{n_{Z} n_{U}}(k)
\end{array}\right] }
\end{aligned}
$$

Assume that the degrees $n_{X}, n_{U}$, and $n_{Z}$ are known and the internal vector $Z(k)$ and the state vector $x(k)$ are 


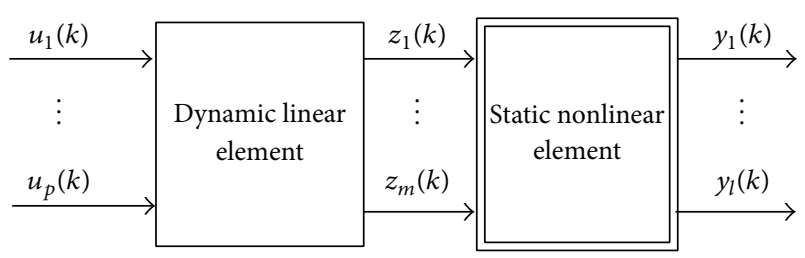

Figure 1: The MIMO Wiener model.

unmeasurable. The static nonlinear function of the MIMO Wiener model is defined as

$$
\begin{aligned}
Y(k)= & f[Z(k)]+E(k) \\
& =\left[\begin{array}{c}
f_{1}\left[z_{1}(k), z_{2}(k), \ldots, z_{n_{Z}}(k)\right] \\
f_{2}\left[z_{1}(k), z_{2}(k), \ldots, z_{n_{Z}}(k)\right] \\
\vdots \\
f_{n_{Y}}\left[z_{1}(k), z_{2}(k), \ldots, z_{n_{Z}}(k)\right]
\end{array}\right] \\
& +\left[\begin{array}{c}
e_{1}(k) \\
e_{2}(k) \\
\vdots \\
e_{n_{Y}}(k)
\end{array}\right],
\end{aligned}
$$

where $Y^{T}(k)=\left[\begin{array}{llll}y_{1}(k) & y_{2}(k) & \cdots & y_{n_{V}}(k)\end{array}\right]$ is the output system vector, $f[Z(k)]$ is the nonlinear function vector that depends on the unknown internal vector $Z(k)$, and $E(k)$ is an error vector.

In the rest of this paper, we propose to rewrite the system output vector $Y(k)$ into two submodels.

The first submodel is given by the following equation:

$$
Y(k)=\Theta \Psi(k, Z(k))+E(k),
$$

where $\Theta$ and $\Psi(k, Z(k))$ are defined by

$$
\begin{aligned}
\Theta & =\left[\begin{array}{ccc}
\theta_{11} & \cdots & \theta_{1 n_{\Psi}} \\
\theta_{21} & \cdots & \theta_{2 n_{\Psi}} \\
\vdots & \ddots & \vdots \\
\theta_{n_{Y} 1} & \cdots & \theta_{n_{Y} n_{\Psi}}
\end{array}\right], \\
\Psi(k, Z(k))= & \left(\begin{array}{c}
\psi_{1}\left(k, z_{1}(k) ; \ldots ; z_{n_{Z}}(k)\right) \\
\psi_{2}\left(k, z_{1}(k) ; \ldots ; z_{n_{Z}}(k)\right) \\
\vdots \\
\psi_{p}\left(k, z_{1}(k) ; \ldots ; z_{n_{Z}}(k)\right)
\end{array}\right) .
\end{aligned}
$$

However, the second submodel is based on a decomposition technique of nonlinear functions $f_{i}[\cdot]$, given in (3):

$$
\begin{aligned}
f_{i} & {\left[z_{1}(k), z_{2}(k), \ldots, z_{n_{Z}}(k)\right] } \\
& =z_{j}(k)+f_{i}^{*}\left[z_{1}(k), z_{2}(k), \ldots, z_{n_{Z}}(k)\right],
\end{aligned}
$$

with $i=1, \ldots, n_{Y}$ and $j=1, \ldots, n_{Z}$.
Using (6), the system output vector $Y(k)$ can be written as

$$
Y(k)=\Gamma Z(k)+\Theta^{\star} \Psi^{\star}(k)+E(k),
$$

where $\Gamma \in R^{n_{Y} \times n_{Z}}$ (if $n_{Y}=n_{Z}, \Gamma=I_{n_{Y} \times n_{Y}} ; I$ is an identity matrix) and $\Theta^{\star} \Psi^{\star}(k)$ is defined by

$$
\begin{aligned}
& \Theta^{\star} \Psi^{\star}(k) \\
& =\left[\begin{array}{ccc}
\theta_{11}{ }^{*} & \cdots & \theta_{1 n_{\Psi}}{ }^{*} \\
\theta_{21}{ }^{*} & \cdots & \theta_{2 n_{\Psi}}{ }^{*} \\
\vdots & \ddots & \vdots \\
\theta_{n_{Y} 1}{ }^{*} & \cdots & \theta_{n_{Y} n_{\Psi}}{ }^{*}
\end{array}\right]\left(\begin{array}{c}
\psi_{1}{ }^{*}\left(k, z_{1}(k) ; \ldots ; z_{n_{Z}}(k)\right) \\
\psi_{2}{ }^{*}\left(k, z_{1}(k) ; \ldots ; z_{n_{Z}}(k)\right) \\
\vdots \\
\psi_{p}{ }^{*}\left(k, z_{1}(k) ; \ldots ; z_{n_{Z}}(k)\right)
\end{array}\right) .
\end{aligned}
$$

It is worth noting that the application of this decomposition technique to Wiener model has led to useful modelisation because it allows the extraction of the inaccessible internal vector $Z(k)$ and then the identification and control schemes become easier and more efficient.

From (1) and (4), it is clear that the number of unknown parameters is quite large, causing difficulties in the identification algorithm implementation. In order to reduce these difficulties and have a precise estimation quality, we propose to estimate the parameters of the linear dynamic part and those of the static nonlinear part, in two separate and recursive steps. The objective of this paper is to present a new recursive method to estimate jointly the parameters $\left(a_{i j}, b_{i j}\right.$, $\left.c_{i j}, d_{i j}\right)$, the state vector $x(k)$, and the intermediate vector $Z(k)$ using the measured input-output data $\{U(k), Y(k)\}$.

\section{The Recursive Parametric and State Estimation Algorithm}

In order to simplify the formulation of the parametric and state estimation problem, this section is divided into two subsections. The basic idea is to estimate recursively the dynamic linear part parameters and the static nonlinear part parameters of the considered MIMO Wiener model and then to estimate the state vector $x(k)$ and the internal vector $Z(k)$.

3.1. The Parameter Estimation Algorithm. If the state vector $x(k)$ and the internal vector $Z(k)$ are known, then we can apply the following adjustable model to generate the linear dynamic subsystem estimate (1):

$$
\begin{aligned}
x_{p}(k+1) & =\widehat{A}(k+1) x(k)+\widehat{B}(k+1) U(k) \\
Z_{p}(k) & =\widehat{C}(k) x(k)+\widehat{D}(k) U(k) .
\end{aligned}
$$

Using the least squares principle and minimizing the prediction errors functions

$$
\begin{aligned}
& \delta_{x}(k)=x(k)-x_{p}(k), \\
& \delta_{Z}(k)=Z(k)-Z_{p}(k),
\end{aligned}
$$


we can obtain the following recursive algorithm:

$$
\begin{aligned}
& \widehat{A}(k)=\widehat{A}(k-1)+\xi_{x}(k-1) G_{x} \delta_{x}(k-1) x^{T}(k-2) \\
& \widehat{B}(k)=\widehat{B}(k-1)+\xi_{x}(k-1) G_{x} \delta_{x}(k-1) U^{T}(k-2) \\
& \widehat{C}(k)=\widehat{C}(k-1)+\xi_{Z}(k-1) G_{Z} \delta_{Z}(k-1) x^{T}(k-1) \\
& \widehat{D}(k) \\
& =\widehat{D}(k-1)+\xi_{Z}(k-1) G_{Z} \delta_{Z}(k-1) U^{T}(k-1) \\
& \delta_{x}(k-1) \\
& =x(k-1)-\widehat{A}(k-1) x(k-2) \\
& -\widehat{B}(k-1) U(k-2) \\
& \delta_{Z}(k-1) \\
& =Z(k-1)-\widehat{C}(k-1) x(k-1) \\
& \text { - } \widehat{D}(k-1) U(k-1) \\
& \xi_{x}(k-1) \\
& =\frac{l_{x}}{\lambda_{G_{x}}\left[x(k-2) x^{T}(k-2)+U(k-2) U^{T}(k-2)\right]} \\
& \xi_{Z}(k-1) \\
& =\frac{l_{Z}}{\lambda_{G_{Z}}\left[x(k-1) x^{T}(k-1)+U(k-1) U^{T}(k-1)\right]},
\end{aligned}
$$

where $G_{x}$ and $G_{Z}$ are definite positive symmetrical matrices and $\lambda_{G_{x}}$ and $\lambda_{G_{Z}}$ are, respectively, the maximum eigenvalues of $G_{x}$ and $G_{Z}$. In order to guarantee the convergence of the parameter estimate matrices $\widehat{A}(k), \widehat{B}(k), \widehat{C}(k)$, and $\widehat{D}(k)$, the gains parameter $l_{x}$ and $l_{z}$ must be chosen such that $0<l_{x}<2$ and $0<l_{Z}<2$. It should be noted that these gains can be chosen as time-varying parameters in order to improve the parameter estimation quality.

To avoid the parametric redundancy problem and construct the estimate parameters of the static nonlinear part (4), we suggest using the following recursive least squares (RLS) algorithm:

$$
\begin{aligned}
& \widehat{\Theta}(k)=\widehat{\Theta}(k-1)+P(k) \Psi(k, Z(k)) \Xi(k) \\
& P(k) \\
& =P(k-1) \\
& -\frac{P(k-1) \Psi(k, Z(k)) \Psi^{T}(k, Z(k)) P(k-1)}{1+\Psi^{T}(k, Z(k)) P(k-1) \Psi(k, Z(k))} \\
& \Xi(k)=Y(k)-\widehat{\Theta}(k-1) \Psi(k, Z(k)),
\end{aligned}
$$

where $\widehat{\Theta}(k)$ represent the estimate of $\Theta$ in the regression equation (4) and $P(k)=p_{0} I_{p \times p}$ is a definite positive symmetrical matrix where $I_{p \times p}$ is an identity matrix and $p_{0}$ is generally taken as a large positive number, for example, $p_{0}=10^{5}$.
In reality, the regression matrix $\Psi(k, Z(k))$ contains the unmeasurable internal vector $Z(k)$, so the parametric estimation algorithms (11) and (12) cannot be implemented directly. Added to that, the state vector $x(k)$ is also supposed to be unknown. The proposed solution is to replace $x(k)$ and $Z(k)$ in (11) and (12) with their estimated vectors $\widehat{x}(k)$ and $\widehat{Z}(k)$ and then to define

$$
\begin{gathered}
\widehat{x}(k)=\left[\begin{array}{c}
\widehat{x}_{1}(k) \\
\widehat{x}_{2}(k) \\
\vdots \\
\widehat{x}_{n_{X}}(k)
\end{array}\right], \\
\widehat{Z}(k)=\left[\begin{array}{c}
\widehat{z}_{1}(k) \\
\widehat{z}_{2}(k) \\
\vdots \\
\widehat{z}_{n_{Z}}(k)
\end{array}\right] .
\end{gathered}
$$

3.2. Modified Kalman Filter. In the area of state estimation algorithms, the extended Kalman filter (EKF) is the most widely used nonlinear estimation method [45]. The EKF is based on the linearization of nonlinear models and calculation of the Jacobian matrices with each iteration, which may cause significant implementation difficulties and high estimation errors. Therefore, we propose to use a new filtering technique, developed in our previous work [15], based on the linear Kalman filter (KF). This filter yields the same performance as KF and can be applied to blocks-oriented models without any linearization steps. Let $\hat{x}(k)$ and $\hat{x}^{0}(k)$ represent, respectively, the a priori and a posteriori estimate of $x(k)$ at a discrete-time $k$ and $\widehat{Z}(k)$ represents the a priori estimate of $Z(k)$.

This part is based essentially on the Kalman filter principle, which is defined by the following.

Theorem 1. Define $\alpha$ and $\beta$ as two random vectors, with $\Lambda=\left[\begin{array}{l}\alpha \\ \beta\end{array}\right]$ a multinomial distribution vector with a mean vector $[\bar{\alpha} \bar{\beta}]$ and a variance-covariance matrix $\left[\begin{array}{cc}\Sigma_{\alpha} & \Sigma_{\alpha \beta} \\ \Sigma_{\alpha \beta}^{T} & \Sigma_{\beta}\end{array}\right]$. Thus, the conditional probability density $p(\alpha, \beta)$ is a Gaussian function, where its mean and variance are given, respectively, by

$$
\begin{aligned}
\widehat{\alpha} & =\bar{\alpha}+\Sigma_{\alpha \beta} \Sigma_{\beta}^{-1}(\beta-\bar{\beta}) \\
\Sigma_{\alpha \backslash \beta} & =\Sigma_{\alpha}-\Sigma_{\alpha \beta} \Sigma_{\beta}^{-1} \Sigma_{\alpha \beta}^{T} .
\end{aligned}
$$

In a first step, applying this theorem to the linear statespace equation (1) yields

$$
\begin{aligned}
& \widehat{x}^{0}(k) \\
& \quad \hat{x}(k) \\
& \quad+K_{x}(k)[Z(k)-\widehat{C}(k) \widehat{x}(k)-\widehat{D}(k) U(k)]
\end{aligned}
$$




$$
\begin{aligned}
& K_{x}(k)=\frac{P_{x}(k) \widehat{C}(k)}{Q_{Z}+\widehat{C}(k) P_{x}(k) \widehat{C}^{T}(k)} \\
& P_{x}^{0}(k)=P_{x}(k)-K_{x}(k) \widehat{C}(k) P_{x}(k) \\
& \widehat{x}(k+1)=\widehat{A}(k) \hat{x}^{0}(k)+\widehat{B}(k) U(k) \\
& P_{x}(k+1)=\widehat{A}(k) P_{x}^{0}(k) \widehat{A}^{T}(k)+Q_{x},
\end{aligned}
$$

where $Q_{x}$ and $Q_{Z}$ are variance-covariance matrices of noise vectors $W(k)$ and $V(k)$, respectively, and $P_{x}(k)=E[(x(k)-$ $\widehat{x}(k))(x(k)-\widehat{x}(k))^{T}$ ] is the variance-covariance matrices.

However, the a posteriori estimate $\hat{x}^{0}(k)$ contains the unknown internal vector $Z(k)$, so the algorithm (15) is impossible to implement. The solution is to replace the unknown internal vector $Z(k)$ with its estimate $\widehat{Z}(k)$. For this reason, we propose to use the second form (7) of the system output vector $Y(k)$ and apply the KF to the following statespace model:

$$
\begin{aligned}
Z(k+1)= & C(k) X(k+1)+D(k) U(k+1) \\
& +W(k+1) \\
Y(k)= & \Gamma Z(k)+\Theta^{\star} \Psi^{\star}(k)+E(k) .
\end{aligned}
$$

Using Theorem 1 gives the following equations to generate the recursive estimate vector $Z(k)$ :

$$
\begin{aligned}
& \widehat{Z}(k+1)=\widehat{C}(k) \widehat{A}(k) \widehat{x}^{0}(k)+\widehat{C}(k) \widehat{B}(k) U(k) \\
& +\widehat{D}(k) U(k+1)+K_{Z}(k)[Y(k)-\Gamma \widehat{C}(k) \hat{x}(k) \\
& \left.-\Gamma \widehat{D}(k) U(k)-\widehat{\Theta}^{\star}(k) \widetilde{\Psi}^{\star}(k)\right] \\
& K_{Z}(k) \\
& =\frac{\widehat{C}(k) \widehat{A}(k) P_{x}(k) \widehat{\Gamma}^{T}(k)}{\Gamma \widehat{C}(k) P_{x}(k) \Gamma \widehat{C}^{T}(k)+Q_{Y}+\widehat{\Theta}^{*}(k) G(k) \widehat{\Theta}^{*^{T}}(k)},
\end{aligned}
$$

where $Q_{Y}$ is the variance-covariance matrix of the noise vector $E(k)$ and $G(k)=E\left[(Y(k)-\widehat{Y}(k))(Y(k)-\widehat{Y}(k))^{T}\right]$ is the variance-covariance matrix.

Combining (15) and (17), we can summarize the recursive algorithm to generate the estimates $\widehat{x}(k)$ and $\widehat{Z}(k)$ of the state vector $x(k)$ and the internal vector $Z(k)$ :

$$
\begin{aligned}
& \widehat{x}^{0}(k)=\widehat{x}(k)+K_{x}(k)[\widehat{Z}(k)-\widehat{C}(k) \widehat{x}(k) \\
& -\widehat{D}(k) U(k)] \\
& K_{x}(k)=\frac{P_{x}(k) \widehat{C}(k)}{Q_{Z}+\widehat{C}(k) P_{x}(k) \widehat{C}^{T}(k)} \\
& P_{x}^{0}(k)=P_{x}(k)-K_{x}(k) \widehat{C}(k) P_{x}(k)
\end{aligned}
$$

$$
\begin{aligned}
& \widehat{x}(k+1)=\widehat{A}(k) \hat{x}^{0}(k)+\widehat{B}(k) U(k) \\
& P_{x}(k+1)=\widehat{A}(k) P_{x}^{0}(k) \widehat{A}^{T}(k)+Q_{x} \\
& \widehat{Z}(k+1)=\widehat{C}(k) \widehat{A}(k) \widehat{x}^{0}(k)+\widehat{C}(k) \widehat{B}(k) U(k) \\
& \quad+\widehat{D}(k) U(k+1)+K_{Z}(k)[Y(k)-\Gamma \widehat{C}(k) \hat{x}(k) \\
& \left.\quad-\Gamma \widehat{D}(k) U(k)-\widehat{\Theta}^{\star}(k) \widetilde{\Psi}^{\star}(k)\right] \\
& K_{Z}(k) \quad \widehat{C}(k) \widehat{A}(k) P_{x}(k) \Gamma \widehat{C}^{T}(k) \\
& \quad=\frac{\Gamma \widehat{C}(k) P_{x}(k) \Gamma \widehat{C}^{T}(k)+Q_{Y}+\widehat{\Theta}^{\star}(k) G(k) \widehat{\Theta}^{\star^{T}}(k)}{} .
\end{aligned}
$$

The details and the convergence analysis of (18) is treated in [15].

Thus, we can replace $x(k)$ and $Z(k)$ in (11) and (12) with their estimates $\widehat{x}(k)$ and $\widehat{Z}(k)$.

Combining (11), (12), and (18), we can form a recursive state and parameter estimation algorithm for state-space MIMO Wiener systems (which is abbreviated as the RPSE algorithm). To initialize the RPSE algorithm, we take $\widehat{x}(0)$, $\widehat{Z}(0), \widehat{A}(0), \widehat{B}(0), \widehat{C}(0), \widehat{D}(0)$, and $\widehat{\Theta}(0)$ as small real matrices and vectors; for example, $\widehat{A}(0)=10^{-3} \imath_{n_{x} \times n_{x}}$ with $\imath_{n_{x} \times n_{x}}$ is a matrix whose all elements are equal to 1 . We also take $G_{x}(k)=$ $g_{x} I, G_{Z}(k)=g_{Z} I, P(k)=p_{0} I, P_{x}(k)=p_{x} I$ with $g_{x}, g_{Z}, p_{0}$, and $p_{x}$ as large positive numbers and $I$ is an identity matrix with appropriate sizes. It should be noted that the choice of the initial conditions and the different gains must be chosen adequately in order to improve the estimation quality and the convergence rapidity of the various parameters.

The procedure for computing the parameter, the state, and the internal vector estimates using the RPSE algorithm is listed as follows:

(1) To initialize, let $k=1, \widehat{x}(1), \widehat{x}(2), \widehat{Z}(1), \widehat{A}(1), \widehat{B}(1)$, $\widehat{C}(1), \widehat{D}(1), \widehat{\Theta}(1), G_{x}, G_{Z}, P(1), l_{x}, l_{Z}, Q_{x}, Q_{Z}, P_{x}(1)$, and $G$.

(2) Collect the input-output vectors $U(k)$ and $Y(k)$.

(3) Form $\xi_{x}(k-1)$ and $\xi_{Z}(k-1)$, compute $\delta_{x}(k-1)$ and $\delta_{Z}(k-1)$, and update $\widehat{A}(k), \widehat{B}(k), \widehat{C}(k), \widehat{D}(k)$ using (11).

(4) Compute the covariance matrix $P(k)$ and construct $\widehat{\Theta}(k)$ using (12).

(5) Compute the state gains $K_{x}(k)$ and $K_{Z}(k)$ and the covariance matrix $P_{x}(k+1)$ and update the state estimate $\hat{x}(k+1)$ and the internal vector estimate $\widehat{Z}(k+1)$ using (18).

(6) Increase $k$ by 1 and go to step (2).

The flowchart of the recursive learning algorithms which are used for the parameters estimation of nonlinear MIMO Wiener models is shown in Figure 2. 


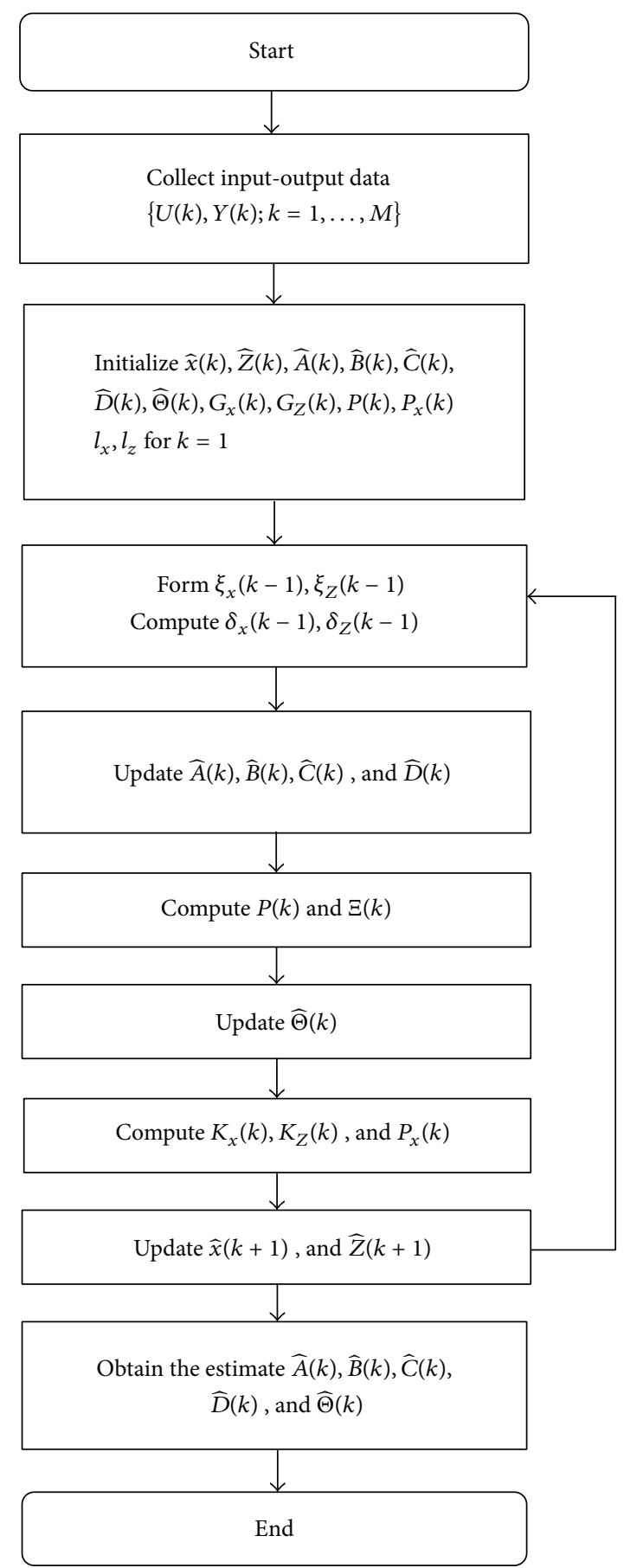

FIGURE 2: Flowchart for recursive estimation of MIMO Wiener models.

\section{Example}

Consider the following state-space model:

$$
\begin{aligned}
x(k+1)= & {\left[\begin{array}{cc}
0 & 0.5 \\
-0.4 & 0
\end{array}\right] x(k) } \\
& +\left[\begin{array}{cc}
0 & \sqrt{0.85} \\
-\sqrt{0.6} & 0
\end{array}\right] U(k)+W(k) \\
Z(k)= & {\left[\begin{array}{ll}
1 & 0 \\
0 & 1
\end{array}\right] x(k)+V(k) }
\end{aligned}
$$

and the system outputs are defined as

$$
\begin{aligned}
& y_{1}(k)=z_{1}(k)+0.3 z_{2}(k)+e_{1}(k) \\
& y_{2}(k)=0.3 z_{1}^{2}(k)+z_{2}(k)+1+e_{2}(k)
\end{aligned}
$$

These two outputs can be grouped into the following matrix form:

$$
Y(k)=\Theta \Psi(k)+E(k),
$$



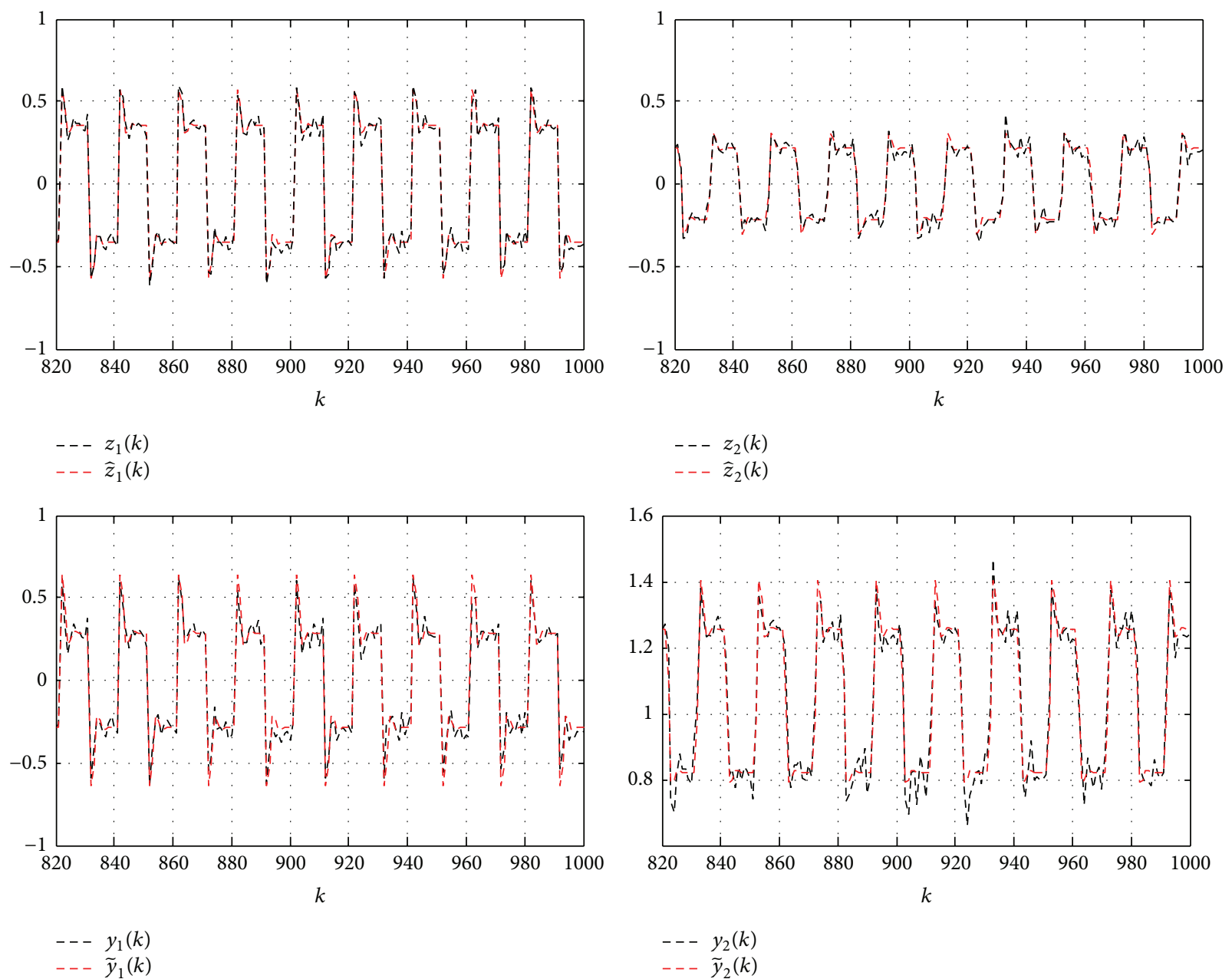

FIGURE 3: The internal variables $z_{1}(k)$ and $z_{2}(k)$ and their estimates $\widehat{z}_{1}(k)$ and $\widehat{z}_{2}(k)$ and the outputs $y_{1}(k)$ and $y_{2}(k)$ with the predicted outputs $\tilde{y}_{1}(k)$ and $\tilde{y}_{2}(k)$.

where $\Theta$ and $\Psi(k)$ are given by

$$
\begin{aligned}
\Theta & =\left[\begin{array}{ccccc}
1 & 0.3 & 0 & 0 & 0 \\
0 & 0 & 0.3 & 1 & 1
\end{array}\right], \\
\Psi^{T}(k) & =\left[\begin{array}{lllll}
z_{1}(k) & z_{2}(k) & z_{1}^{2}(k) & z_{2}(k) & 1
\end{array}\right]
\end{aligned}
$$

Using the decomposition technique, (21) can be written in a second matrix form:

$$
Y(k)=Z(k)+\Theta^{*} \Psi^{*}(k)+E(k),
$$

where $\Theta^{*}$ and $\Psi^{*}(k)$ are defined as

$$
\begin{aligned}
\Theta^{*} & =\left[\begin{array}{ccc}
0 & 0.3 & 0 \\
0.3 & 0 & 1
\end{array}\right] \\
\Psi^{* T}(k) & =\left[\begin{array}{lll}
z_{1}^{2}(k) & z_{2}(k) & 1
\end{array}\right]
\end{aligned}
$$

In simulation, the inputs $u_{1}(k)$ and $u_{2}(k)$ are taken as two square sequences of levels $\left[\begin{array}{ll}-1.2 & 1.2\end{array}\right]$ and $\left[\begin{array}{ll}-1.5 & 1.5\end{array}\right]$, respectively; the variance-covariance matrices are $Q_{x}=$ $\left[\begin{array}{cc}0.005 & 0 \\ 0 & 0.004\end{array}\right], Q_{Z}=\left[\begin{array}{cc}0.015 & 0 \\ 0 & 0.015\end{array}\right]$, and $Q_{Y}=\sigma_{e}^{2}\left[\begin{array}{ll}1 & 0 \\ 0 & 1\end{array}\right]$. Applying the RPSE algorithm to estimate the parameters, the state, and internal variables of this system, the gain parameters and the initial conditions are chosen properly. The internal variables $z_{1}(k)$ and $z_{2}(k)$ and their estimates $\widehat{z}_{1}(k)$ and $\widehat{z}_{2}(k)$ and the outputs $y_{1}(k)$ and $y_{2}(k)$ with the predicted outputs $\tilde{y}_{1}(k)$ and $\tilde{y}_{2}(k)$ are shown in Figure 3 . The estimation errors $\delta_{z_{1}}(k), \delta_{z_{2}}(k), \delta_{y_{1}}(k)$, and $\delta_{y_{2}}(k)$ are shown in Figure 4. The evolution curve of the variance $\sigma_{y_{1}}{ }^{2}(k)$ and $\sigma_{y_{2}}{ }^{2}(k)$ of the system outputs $y_{1}(k)$ and $y_{2}(k)$ is given in Figure 5, where

$$
\sigma_{y_{i}}{ }^{2}(k)=\frac{\Sigma\left[\delta_{y_{i}}(k)-\bar{m}_{\delta_{y_{i}}}\right]^{2}}{k_{f}-k_{i}+1},
$$

with $\bar{m}_{\delta_{y_{j}}}$ the statistical mean of the output prediction error and $k_{i}$ and $k_{f}$ the initial and final discrete time. The parameter estimates and their estimation errors with different data length and different noise variances are shown in Tables 1 and 2 , where the parameter estimation error is defined by 

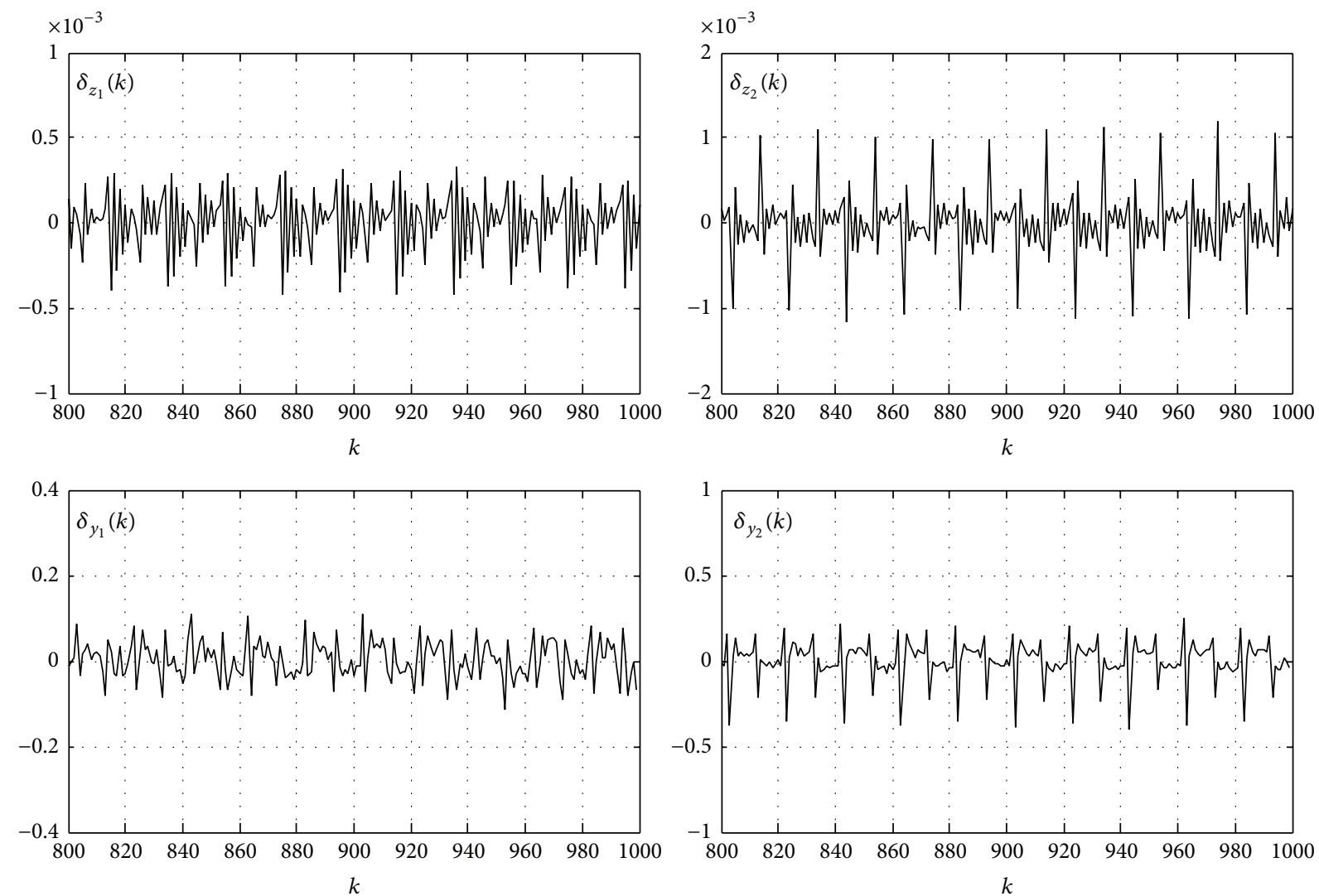

FIGURE 4: The estimation errors $\delta_{z_{1}}(k), \delta_{z_{2}}(k), \delta_{y_{1}}(k)$, and $\delta_{y_{2}}(k)$.

$\delta(k)$

$=\left[\frac{\left[\sum_{i=1}^{2}\left[\sum_{j=1}^{2}\left(a_{i j}-\widehat{a}_{i j}(k)\right)^{2}\right]+\sum_{i=1}^{2}\left[\sum_{j=1}^{2}\left(b_{i j}-\widehat{b}_{i j}(k)\right)^{2}\right]+\sum_{i=1}^{2}\left[\sum_{j=1}^{2}\left(c_{i j}-\widehat{c}_{i j}(k)\right)^{2}\right]+\sum_{r=1}^{5}\left[\sum_{s=1}^{2}\left(\theta_{r s}-\widehat{\theta}_{r s}(k)\right)^{2}\right]\right]}{\left[\sum_{i=1}^{2} \sum_{j=1}^{2}\left(a_{i j}\right)^{2}+\sum_{i=1}^{2} \sum_{j=1}^{2}\left(b_{i j}\right)^{2}+\sum_{i=1}^{2} \sum_{j=1}^{2}\left(c_{i j}\right)^{2}+\sum_{r=1}^{2} \sum_{s=1}^{2}\left(\theta_{r s}\right)^{2}\right]}\right]$

$\times 100$.

From the simulation results in Tables 1, 2, and 3 and Figures 3, 4, 5, and 6, we can draw the following conclusions:

(i) The estimated parameters converge to real ones and the parameter estimation errors given by the RPSE algorithm become smaller when $k$ increases and the output variances decrease; see Tables 1 and 2.

(ii) The estimated internal outputs $\widehat{z}_{1}(k)$ and $\widehat{z}_{2}(k)$ and the predicted system outputs $\tilde{y}_{1}(k)$ and $\tilde{y}_{2}(k)$ can track the actual outputs without the computation step of the inverse nonlinear function and with small estimation errors; see Figures 3 and 4.

(iii) The output variances $\sigma_{y_{1}}{ }^{2}(k)$ and $\sigma_{y_{2}}{ }^{2}(k)$ rapidly drop to law values when the noise variances decrease; see Figure 5. (iv) The estimation quality is better when the parametric gains $l_{x}$ and $l_{Z}$ are chosen as a time-varying parameters; see Figure 6.

(v) The variances of the parameters estimates, using the Monte Carlo simulation, are small which improves the effectiveness of the RPSE algorithm; see Table 3.

(vi) The proposed algorithm can achieve a satisfactory estimation quality through appropriately choosing the parametric gains and the innovation length.

\section{Conclusions}

This paper presents a recursive parameter and state estimation algorithm by combining the least square technique, the adjustable model, and the Kalman filter principal for 


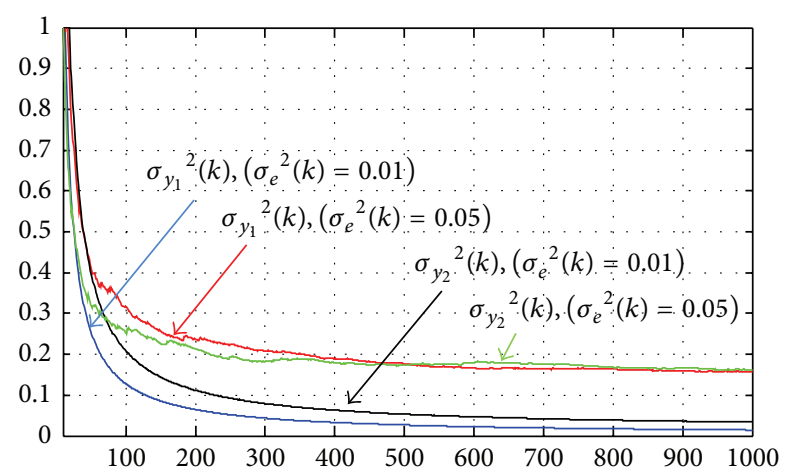

FIGURE 5: The output variances $\sigma_{y_{1}}{ }^{2}(k)$ and $\sigma_{y_{2}}{ }^{2}(k)$ with $\sigma_{e}{ }^{2}=0.01$ and $\sigma_{e}^{2}=0.05$.

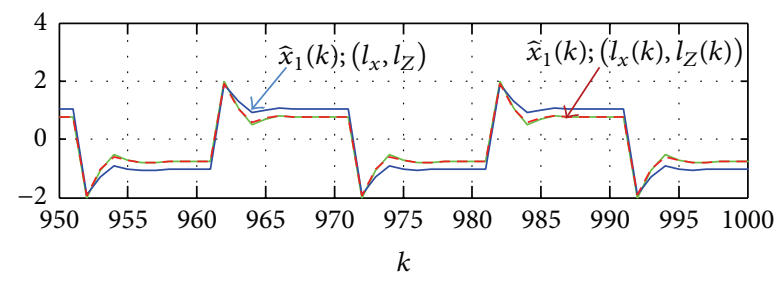

$-x_{1}(k)$

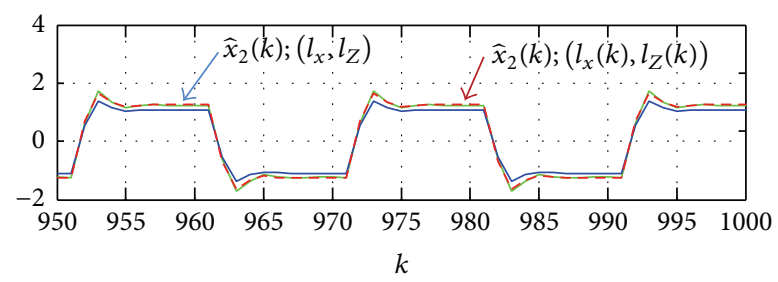

$-x_{2}(k)$

Figure 6: The state estimates $\widehat{x}_{1}(k)$ and $\widehat{x}_{2}(k)$ with constant gains $\left(l_{x}, l_{Z}\right)$ and variable parametric gains $\left(l_{x}(k), l_{Z}(k)\right)$.

estimating jointly the parameters, the state vector, and the internal variables of MIMO Wiener state-space models. By estimating the parameters of the linear and nonlinear parts separately and using a specific decomposition technique, we can remove the redundant parameters and avoid problems related to computing the inverse nonlinear functions. The proposed algorithm can be combined with adaptive control schemes and extended to other blocks-oriented models.

\section{Competing Interests}

The authors declare that there are no competing interests regarding the publication of this paper.

\section{Acknowledgments}

This work was supported by the ministry of higher education and scientific research of Tunisia.
TABLE 1: The recursive parameter estimates and errors with $\sigma_{e}^{2}=$ 0.01 .

\begin{tabular}{lcccc}
\hline$M$ & 100 & 200 & 500 & 1000 \\
\hline$a_{11}(k)=0.0000$ & -0.0166 & -0.0008 & -0.0002 & -0.0001 \\
$a_{12}(k)=0.5000$ & 0.4769 & 0.4989 & 0.5000 & 0.5001 \\
$a_{21}(k)=-0.4000$ & -0.3965 & -0.3998 & -0.4000 & -0.4000 \\
$a_{22}(k)=0.0000$ & 0.0048 & 0.0002 & 0.0001 & 0.0000 \\
$b_{11}(k)=0.0000$ & 0.001 & 0.0022 & 0.0001 & 0.0000 \\
$b_{12}(k)=0.9220$ & 0.8976 & 0.8991 & 0.9108 & 0.9219 \\
$b_{21}(k)=-0.7746$ & -0.2654 & -0.5653 & -0.7731 & -0.7744 \\
$b_{22}(k)=0.0000$ & -0.1022 & 0.0101 & 0.0105 & -0.0002 \\
$c_{11}(k)=1.0000$ & 1.0012 & 1.0000 & 1.0000 & 1.0000 \\
$c_{12}(k)=0.0000$ & 0.0019 & 0.0000 & 0.0000 & 0.0000 \\
$c_{21}(k)=0.0000$ & -0.0007 & -0.0000 & -0.0000 & -0.0000 \\
$c_{22}(k)=1.0000$ & 0.9989 & 1.0000 & 1.0000 & 1.0000 \\
$\theta_{11}(k)=1.0000$ & 0.9573 & 0.9902 & 1.0027 & 1.000 \\
$\theta_{12}(k)=0.3000$ & 0.1502 & 0.1853 & 0.2889 & 0.3001 \\
$\theta_{13}(k)=0.0000$ & 0.0167 & -0.0027 & 0.0016 & 0.0010 \\
$\theta_{14}(k)=0.0000$ & 0.1202 & 0.1153 & 0.1108 & 0.0979 \\
$\theta_{15}(k)=0.0000$ & 0.0027 & 0.00620 & 0.0015 & 0.0001 \\
$\theta_{21}(k)=0.0000$ & -0.0793 & -0.0046 & 0.0003 & 0.0000 \\
$\theta_{22}(k)=0.0000$ & 0.0036 & 0.0043 & 0.0014 & 0.0002 \\
$\theta_{23}(k)=0.3000$ & 0.2306 & 0.2490 & 0.2913 & 0.3001 \\
$\theta_{24}(k)=1.0000$ & 0.3797 & 0.5472 & 0.9894 & 0.9989 \\
$\theta_{25}(k)=1.0000$ & 0.9941 & 0.9526 & 1.0008 & 1.0001 \\
$\delta(k)(\%)$ & 31.6697 & 19.9767 & 4.2708 & 3.6902 \\
\hline & & & &
\end{tabular}

TABLE 2: The recursive parameter estimates and errors with $\sigma_{e}^{2}=$ 0.05 .

\begin{tabular}{lcccc}
\hline$M$ & 100 & 200 & 500 & 1000 \\
\hline$a_{11}(k)=0.0000$ & -0.0166 & -0.0008 & 0.0000 & 0.0000 \\
$a_{12}(k)=0.5000$ & 0.4769 & 0.4989 & 0.5002 & 0.5000 \\
$a_{21}(k)=-0.4000$ & -0.3965 & -0.3998 & -0.4000 & -0.4000 \\
$a_{22}(k)=0.0000$ & 0.0048 & 0.0002 & 0.0000 & 0.0001 \\
$b_{11}(k)=0.0000$ & 0.0012 & 0.0011 & 0.0010 & 0.0010 \\
$b_{12}(k)=0.9220$ & 0.8974 & 0.8996 & 0.8999 & 0.9105 \\
$b_{21}(k)=-0.7746$ & -0.2654 & -0.5651 & -0.7659 & -0.7699 \\
$b_{22}(k)=0.0000$ & -0.1022 & -0.0101 & -0.0101 & -0.0091 \\
$c_{11}(k)=1.0000$ & 1.0012 & 1.0000 & 1.0000 & 1.0000 \\
$c_{12}(k)=0.0000$ & 0.0019 & 0.0000 & 0.0000 & 0.0000 \\
$c_{21}(k)=0.0000$ & -0.0007 & 0.0000 & 0.0000 & 0.0000 \\
$c_{22}(k)=1.0000$ & 0.9989 & 0.9995 & 1.0000 & 1.0000 \\
$\theta_{11}(k)=1.0000$ & 0.9071 & 0.9567 & 1.0082 & 0.9976 \\
$\theta_{12}(k)=0.3000$ & 0.1710 & 0.1773 & 0.2705 & 0.2995 \\
$\theta_{13}(k)=0.0000$ & 0.0123 & -0.0052 & 0.0013 & 0.001 \\
$\theta_{14}(k)=0.0000$ & 0.1410 & 0.0473 & 0.1205 & 0.0992 \\
$\theta_{15}(k)=0.0000$ & -0.0597 & 0.00731 & -0.00384 & -0.0025 \\
$\theta_{21}(k)=0.0000$ & -0.0016 & -0.0075 & -0.0010 & 0.0013 \\
$\theta_{22}(k)=0.0000$ & 0.0044 & 0.0044 & 0.0014 & 0.0001 \\
$\theta_{23}(k)=0.3000$ & 0.4817 & 0.3529 & 0.2921 & 0.2997 \\
$\theta_{24}(k)=1.0000$ & 0.4528 & 0.5773 & 0.9541 & 0.9903 \\
$\theta_{25}(k)=1.0000$ & 0.9679 & 0.9486 & 1.0023 & 1.0010 \\
$\delta(k)(\%)$ & 31.4375 & 18.7664 & 5.1010 & 3.8043 \\
\hline
\end{tabular}




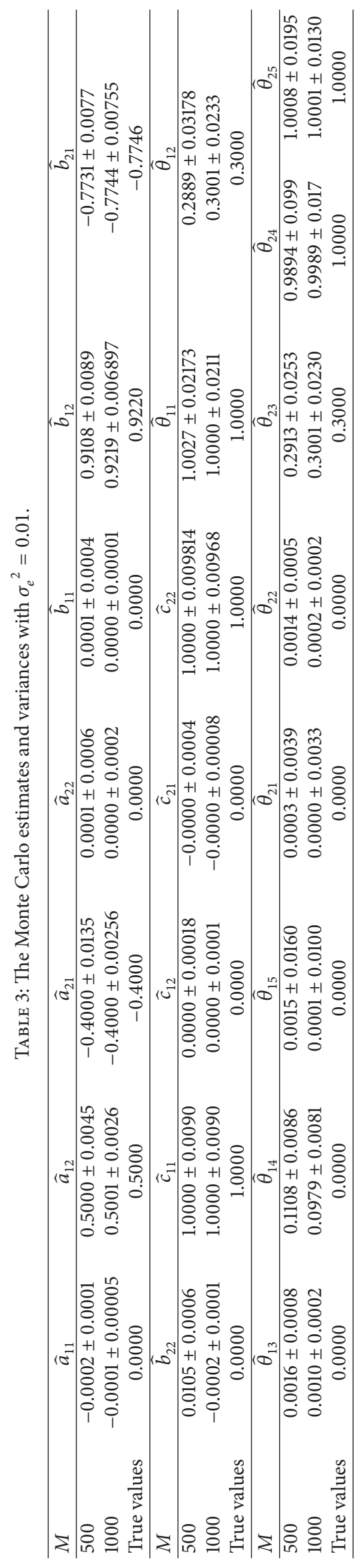




\section{References}

[1] H. Han, L. Xie, F. Ding, and X. Liu, "Hierarchical least-squares based iterative identification for multivariable systems with moving average noises," Mathematical and Computer Modelling, vol. 51, no. 9-10, pp. 1213-1220, 2010.

[2] R. Q. Fuentes, I. Chairez, A. Poznyak, and T. Poznyak, "3D nonparametric neural identification," Journal of Control Science and Engineering, vol. 2012, Article ID 618403, 10 pages, 2012.

[3] F. Ding, L. Qiu, and T. Chen, "Reconstruction of continuoustime systems from their non-uniformly sampled discrete-time systems," Automatica, vol. 45, no. 2, pp. 324-332, 2009.

[4] Y. Liu, F. Ding, and Y. Shi, "An efficient hierarchical identification method for general dual-rate sampled-data systems," Automatica, vol. 50, no. 3, pp. 962-970, 2014.

[5] A. Fathi and A. Mozaffari, "Identification of a dynamic model for shape memory alloy actuator using Hammerstein-Wiener gray box and mutable smart bee algorithm," International Journal of Intelligent Computing and Cybernetics, vol. 6, no. 4, pp. 328-357, 2013.

[6] A. D. Kalafatis, L. Wang, and W. R. Cluett, "Linearizing feedforward-feedback control of $\mathrm{pH}$ processes based on the Wiener model," Journal of Process Control, vol. 15, no. 1, pp. 103$112,2005$.

[7] A. Bhattacharjee and A. Sutradhar, "Online identification and internal model control for regulating hemodynamic variables in congestive heart failure patient," International Journal of Pharma Medicine and Biological Sciences, vol. 4, no. 2, pp. 85-89, 2015.

[8] Y. Mao and F. Ding, "Multi-innovation stochastic gradient identification for Hammerstein controlled autoregressive autoregressive systems based on the filtering technique," Nonlinear Dynamics, vol. 79, no. 3, pp. 1745-1755, 2015.

[9] W. Yu, D. Wilson, and B. Young, "Control performance assessment for block-oriented nonlinear systems," in Proceedings of the 8th IEEE International Conference on Control and Automation (ICCA '10), pp. 1151-1156, Xiamen, China, June 2010.

[10] S. I. Biagiola and J. L. Figueroa, "Wiener and Hammerstein uncertain models identification," Mathematics and Computers in Simulation, vol. 79, no. 11, pp. 3296-3313, 2009.

[11] F. Guo, A new identification method for wiener and hammerstein systems [Ph.D. thesis], Karlsruhe University, 2004.

[12] M. Salimifard, M. Jafari, and M. Dehghani, "Identification of nonlinear MIMO block-oriented systems with moving average noises using gradient based and least squares based iterative algorithms," Neurocomputing, vol. 94, pp. 22-31, 2012.

[13] H. Salhi, S. Kamoun, N. Essounbouli, and A. Hamzaoui, "Adaptive discrete-time sliding-mode control of nonlinear systems described by Wiener models," International Journal of Control, vol. 89, no. 3, pp. 611-622, 2016.

[14] H. Salhi and S. Kamoun, "A recursive parametric estimation algorithm of multivariable nonlinear systems described by Hammerstein mathematical models," Applied Mathematical Modelling Journal, vol. 39, no. 16, pp. 4951-4962, 2015.

[15] H. Salhi and S. Kamoun, "State and parametric estimation of nonlinear systems described by wiener sate-space mathematical models," in Handbook of Research on Advanced Intelligent Control Engineering and Automation, chapter 4, pp. 107-147, 2014.

[16] S. I. Biagiola and J. L. Figueroa, "Identification of uncertain MIMO Wiener and Hammerstein models," Computers and Chemical Engineering, vol. 35, no. 12, pp. 2867-2875, 2011.
[17] S. Lakshminarayanan, S. L. Shah, and K. Nandakumar, "Identification of Hammerstein models using multivariate statistical tools," Chemical Engineering Science, vol. 50, no. 22, pp. 35993613, 1995.

[18] F. Ding, "Hierarchical multi-innovation stochastic gradient algorithm for Hammerstein nonlinear system modeling," Applied Mathematical Modelling, vol. 37, no. 4, pp. 1694-1704, 2013.

[19] Z. Zhang, F. Ding, and X. Liu, "Hierarchical gradient based iterative parameter estimation algorithm for multivariable output error moving average systems," Computers \& Mathematics with Applications, vol. 61, no. 3, pp. 672-682, 2011.

[20] J. Vörös, "Iterative identification of nonlinear dynamic systems with output backlash using three-block cascade models," Nonlinear Dynamics, vol. 79, no. 3, pp. 2187-2195, 2015.

[21] Y. Hu, B. Liu, Q. Zhou, and C. Yang, "Recursive extended least squares parameter estimation for Wiener nonlinear systems with moving average noises," Circuits, Systems, and Signal Processing, vol. 33, no. 2, pp. 655-664, 2014.

[22] F. Ding, X. G. Liu, and J. Chu, "Gradient-based and leastsquares-based iterative algorithms for Hammerstein systems using the hierarchical identification principle," IET Control Theory \& Applications, vol. 7, no. 2, pp. 176-184, 2013.

[23] J. H. Li, "Parameter estimation for Hammerstein CARARMA systems based on the Newton iteration," Applied Mathematics Letters, vol. 26, no. 1, pp. 91-96, 2013.

[24] F. Guo and G. Bretthauer, "Identification of MISO Wiener and Hammerstein systems," in Proceedings of the 7th European Control Conference (TEE '03), pp. 2144-2149, University of Cambridge, September 2003.

[25] N. I. Chaudhary, M. A. Z. Raja, J. A. Khan, and M. S. Aslam, "Identification of input nonlinear control autoregressive systems using fractional signal processing approach," The Scientific World Journal, vol. 2013, Article ID 467276, 13 pages, 2013.

[26] Z. Wu, S. Peng, B. Chen, and H. Zhao, "Robust Hammerstein adaptive filtering under maximum correntropy criterion," Entropy, vol. 17, no. 10, pp. 7149-7166, 2015.

[27] T. Falck, P. Dreesen, K. De Brabanter, K. Pelckmans, B. De Moor, and J. A. K. Suykens, "Least-squares support vector machines for the identification of Wiener-Hammerstein systems," Control Engineering Practice, vol. 20, no. 11, pp. 1165-1174, 2012.

[28] A. Y. Kibangou and G. Favier, "Identification of parallelcascade Wiener systems using joint diagonalization of thirdorder Volterra kernel slices," IEEE Signal Processing Letters, vol. 16, no. 3, pp. 188-191, 2009.

[29] A. Y. Kibangou and G. Favier, “Tensor analysis-based model structure determination and parameter estimation for blockoriented nonlinear systems," IEEE Journal on Selected Topics in Signal Processing, vol. 4, no. 3, pp. 514-525, 2010.

[30] X. Wang and F. Ding, "Modelling and multi-innovation parameter identification for Hammerstein nonlinear state space systems using the filtering technique," Mathematical and Computer Modelling of Dynamical Systems, vol. 22, no. 2, pp. 113-140, 2016.

[31] F. Ding, X. Liu, and X. Ma, "Kalman state filtering based least squares iterative parameter estimation for observer canonical state space systems using decomposition," Journal of Computational and Applied Mathematics, vol. 301, pp. 135-143, 2016.

[32] F. Ding, "Combined state and least squares parameter estimation algorithms for dynamic systems," Applied Mathematical Modelling, vol. 38, no. 1, pp. 403-412, 2014. 
[33] X. Ma and F. Ding, "Gradient-based parameter identification algorithms for observer canonical state space systems using state estimates," Circuits, Systems, and Signal Processing, vol. 34, no. 5, pp. 1697-1709, 2015.

[34] F. Ding and T. Chen, "Hierarchical identification of lifted statespace models for general dual-rate systems," IEEE Transactions on Circuits and Systems I: Regular Papers, vol. 52, no. 6, pp. 11791187, 2005.

[35] X. Ma and F. Ding, "Recursive and iterative least squares parameter estimation algorithms for observability canonical state space systems," Journal of the Franklin Institute. Engineering and Applied Mathematics, vol. 352, no. 1, pp. 248-258, 2015.

[36] X. Wang and F. Ding, "Recursive parameter and state estimation for an input nonlinear state space system using the hierarchical identification principle," Signal Processing, vol. 117, pp. 208-218, 2015.

[37] D.-Q. Wang and F. Ding, "Hierarchical least squares estimation algorithm for hammerstein-wiener systems," IEEE Signal Processing Letters, vol. 19, no. 12, pp. 825-828, 2012.

[38] D. Wang, F. Ding, and L. Ximei, "Least squares algorithm for an input nonlinear system with a dynamic subspace state space model," Nonlinear Dynamics, vol. 75, no. 1-2, pp. 49-61, 2014.

[39] D. Westwick and M. Verhaegen, "Identifying MIMO Wiener systems using subspace model identification methods," Signal Processing, vol. 52, no. 2, pp. 235-258, 1996.

[40] J. Bruls, C. T. Chou, B. R. J. Haverkamp, and M. Verhaegen, "Linear and non-linear system identification using separable least-squares," European Journal of Control, vol. 5, no. 1, pp. 116128, 1999.

[41] M. Lovera, T. Gustafsson, and M. Verhaegen, "Recursive subspace identification of linear and non-linear Wiener state-space models," Automatica, vol. 36, no. 11, pp. 1639-1650, 2000.

[42] T. Á. Glaria Lopez and D. Sbarbaro, "Observer design for nonlinear processes with Wiener structure," in Proceedings of the 50th IEEE Conference on Decision and Control and European Control Conference (CDC-ECC '11), pp. 2311-2316, IEEE, Orlando, Fla, USA, December 2011.

[43] E.-W. Bai, "Identification of linear systems with hard input nonlinearities of known structure," Automatica, vol. 38, no. 5, pp. 853-860, 2002.

[44] J. Vörös, "Parameter identification of Wiener systems with discontinuous nonlinearities," Systems and Control Letters, vol. 44, no. 5, pp. 363-372, 2001.

[45] K. Xiong, C. L. Wei, and L. D. Liu, "Robust Kalman filtering for discrete-time nonlinear systems with parameter uncertainties," Aerospace Science and Technology, vol. 18, no. 1, pp. 15-24, 2012. 


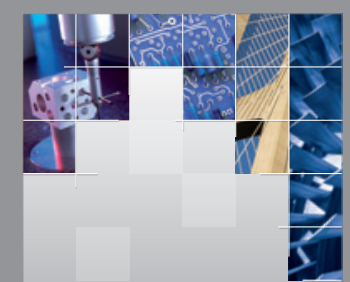

\section{Enfincering}
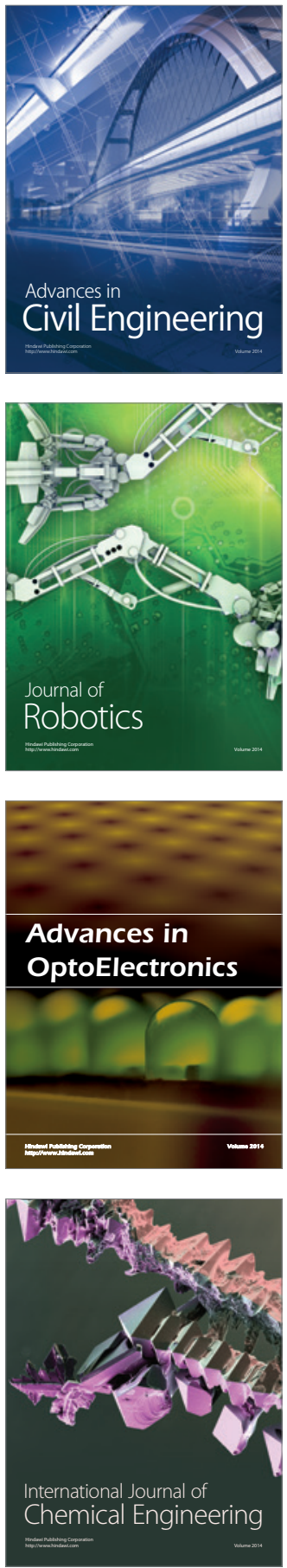

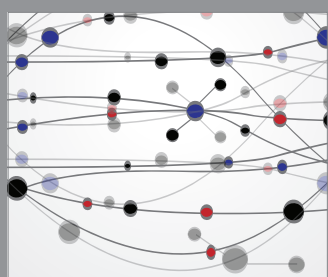

The Scientific World Journal

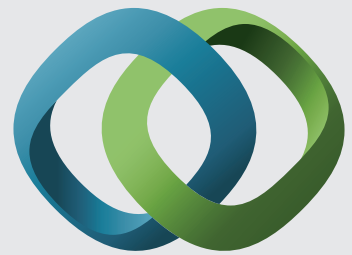

\section{Hindawi}

Submit your manuscripts at

http://www.hindawi.com
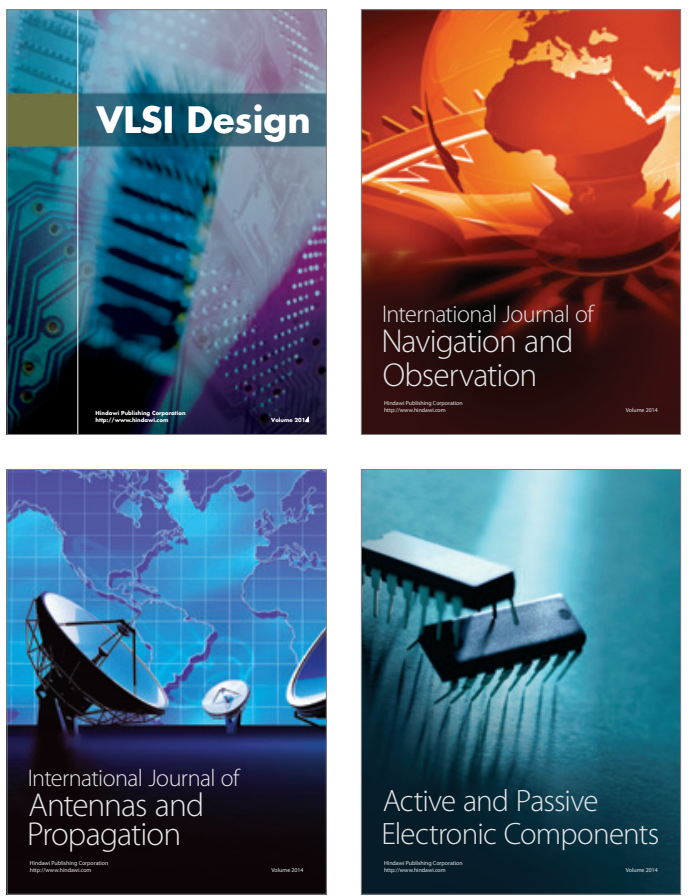
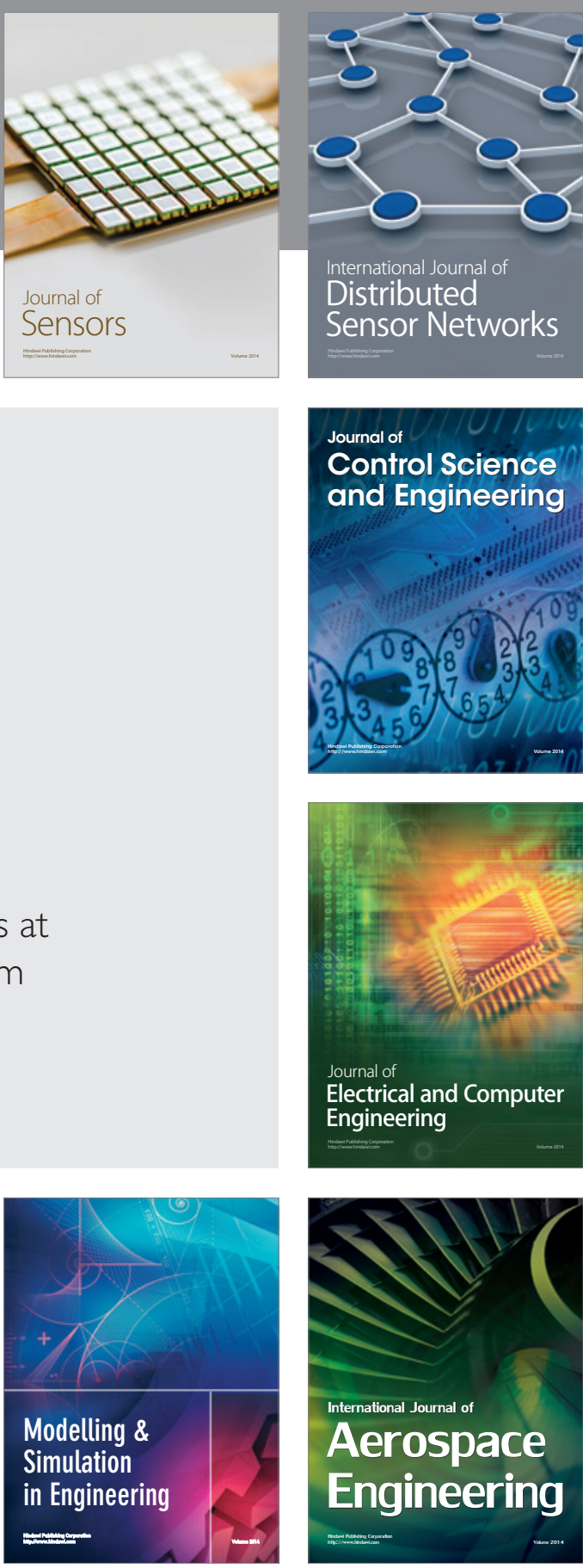

International Journal of

Distributed

Sensor Networks

Journal of

Control Science

and Engineering
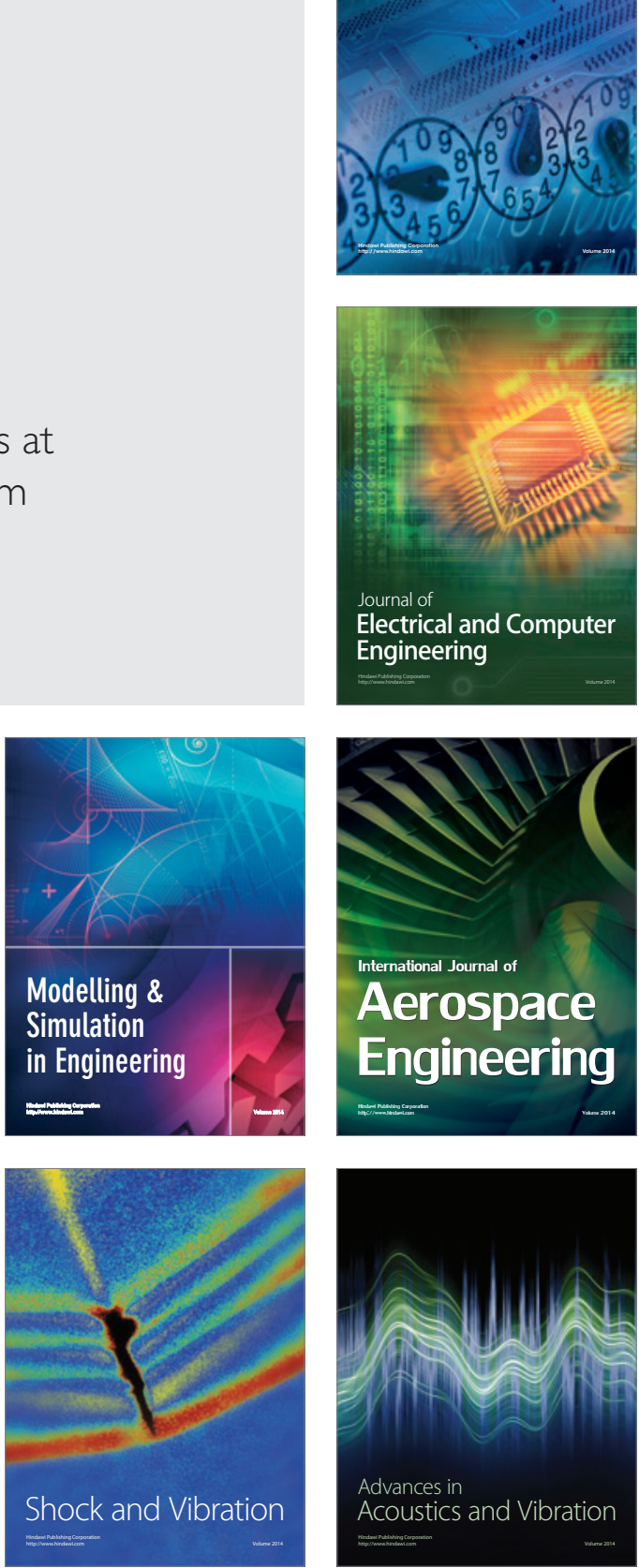